\title{
The Quality of Sub-daily Polar Motion Estimates based on GPS Observations
}

\author{
R. Weber \\ Department of Advanced Geodesy, University of Technology Vienna, \\ Gusshausstrasse 27-29, Vienna, A-1040, Austria \\ M. Rothacher \\ Astronomical Institute, University of Bern, Sidlerstrasse 5, CH-3012 \\ Bern, Switzerland
}

\begin{abstract}
Since June 1992 the International GPS Service (IGS) has provided time series of its products comprising, among others, very accurate polar motion estimates. While the products of the pilot phase were based on data from about 30 permanent stations, the network now consists of about 140 globally distributed tracking sites (Neilan, 1998).

Several efforts have been undertaken at the CODE Analysis Center of the IGS aiming at the optimization of the global processing strategy, including satellite orbit and troposphere modeling. In addition, the time resolution of the ERP series has been increased to two hours or even one hour. This paper deals with sub-daily polar motion and LOD series of four months in late 1996, which have been calculated in a reprocessing step very recently. The investigations performed allow for comparisons of the new series with results stemming from intensive VLBI tracking campaigns and for the detailed study of the influence of the processing strategy and parametrization on the ERP series.
\end{abstract}

\section{Introduction}

GPS data has proven to be a valuable source for the computation of highly accurate Earth Rotation Parameter series (ERPs). This statement holds especially for the period from 1993 till 1998, because (compared to other techniques) of the uninterrupted coverage with observation data and the more-or-less regular distribution of the active tracking sites.

On the other hand, due to ongoing attempts to improve the processing strategy, the resulting series sometimes suffer from inhomogenity and are subsequently difficult to interpret (see e.g. Rothacher et al., 1998). At the CODE Analysis Center, for example, several model changes concerning the force field, the weighting of the observations (elevation dependence), the time resolution of the estimates or the realization of the reference frame can be reported from 1993 to 1998 . Thus, the homogeneous recalculation of the ERP series with the most recent (and best available) strategy promises a remarkable reduction of inherent systematic errors. If the interesting parameters are already part of the saved daily normal equations, this effort seems to be feasible very quickly. In 
our case, caused by the increased time resolution of $1 \mathrm{~h}$, the results have to be generated by reprocessing a huge amount of raw data, starting from "cleaned" single difference files. In the long run, our motivation is to prepare a state-ofthe-art ERP series covering the period 1993-1996, because this span obviously suffers most from a sub-optimal modeling of the GPS data up to now.

\section{Character of the Solution}

The interval of our test calculations started on Sept. 1, 1996 and ended on Dec. 31 , of the same year. In order to realize the terrestrial system 37 stations were fixed to their ITRF96 coordinates. Solid Earth tides were modeled according to the IERS conventions 1996 (McCarthy, 1996) and three dimensional site displacements due to ocean tide loading have been considered by means of Scherneck's ocean model coefficients (Scherneck, 1991).

Troposphere zenith path delays were estimated every 6 hours, i.e. 4 parameters were determined per site and day. These zenith delays were mapped to the actual zenith angles of the satellites by means of the Saastamoinen function. $10^{\circ}$ was chosen as cut-off angle and a height-dependent weighting of the observations was applied. The large number of stations (and with that a remarkable number of unknowns) prevents further reduction of the binning interval for troposphere parameters. This might be one of the accuracy limiting factors for our ERP estimates, because the validity interval of 6 hours is almost commensurate with semidiurnal and diurnal tidal periods.

The orbits are mainly characterized by one set of initial conditions per satellite as well as an "extended" radiation pressure model consisting of three constant terms along the axis of a coordinate system attached to the spacecraft (direct, $\mathrm{y}$-bias, $\mathrm{x}$-bias) and two periodic coefficients per revolution along the $\mathrm{x}$ axis (Beutler et al., 1994). Additionally small pseudo-stochastic pulses are set up in the along-track and radial directions. The dynamical effect of ocean tides is considered (Eanes et al., 1996).

As mentioned above, sub-daily ERPs were computed with respect to the model by Ray (McCarthy, 1996) with a time resolution of one hour. Because of the well-known effect, that satellite techniques are restricted to estimate UT rates, the difference UT-UTC has to be recovered by integration. Nutation offset drift parameters were constrained to reflect the IERS 1996 Theory of Nutation.

\section{Initial Results}

Focusing on the rotation component, the series obtained contain besides the expected differences in the diurnal and semidiurnal band some unexpected peaks at $8,6,4.8,4,3, \ldots$ hours (at the higher order harmonics of 1 cyc/day) with decreasing amplitudes. A kind of leakage effect would be a reasonable explanation. In addition, we want to call attention to the small peak at 5.15 hours showing up in the "power spectrum" in Figure 1, which cannot be explained in a similar way.

A short look at the residual "amplitude spectrum" in polar motion shows us large, differences remaining in the prograde diurnal band (Figure 2). Due to the short series, a more detailed assignment to special waves is not possible at 


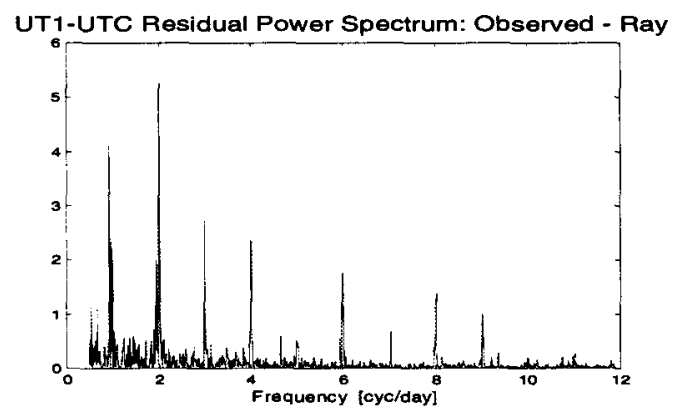

Figure 1. Residual power spectrum.

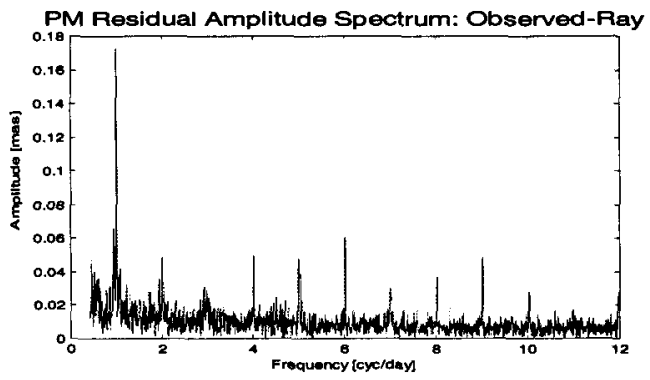

Figure 2. Prograde polar motion.

the moment. But it might be assumed, that the differences stem from tides with periods very close to 24 hours $\left(S_{1}, \psi_{1}\right)$ and therefore within the $2: 1$ resonance of the orbit period and the sidereal day.

Similar to the UT series there are small peaks at the higher harmonics of one cycle/day, which have to be investigated in the near future. Figure 3 shows the corresponding retrograde spectrum (same scale) giving evidence that terms at the nutation frequency have been suppressed succesfully. Moreover, both plots tell us that GPS results and the Ray model correspond fairly well in the semidiurnal band.

Unfortunately uninterrupted VLBI-based sub-daily ERPs are not available for the whole period. Therefore Figure 4 shows a blow-up of 12 days in September 1996. UT variations of both techniques had systematic drifts removed and depicted together with the corresponding values of the evaluated Ray model.

The rms agreement of the GPS estimates with the Ray model calculated for the plotted span is at the $\pm 12 \mu s$ and $\pm 100 \mu a s$ level, which is consistent within 3times the formal errors of the estimates and the precision of the reference model. GPS and VLBI ERP series agree at about the same level in UT and slightly 


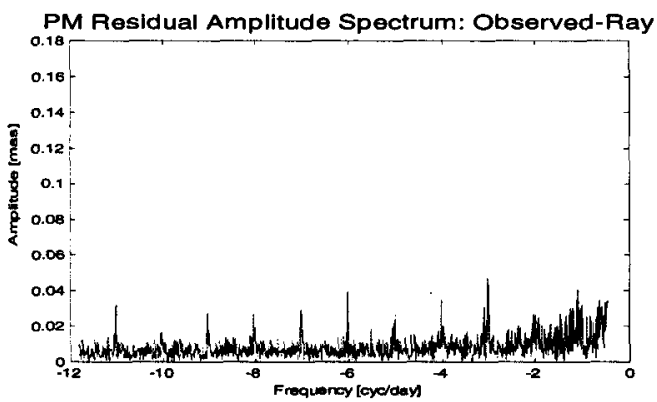

Figure 3. Retrograde polar motion.

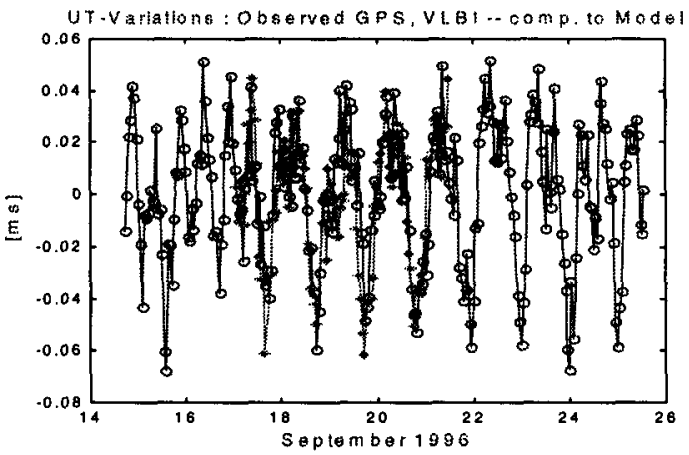

Figure 4. GPS (circles), VLBI (stars), Ray model (solid-line). 


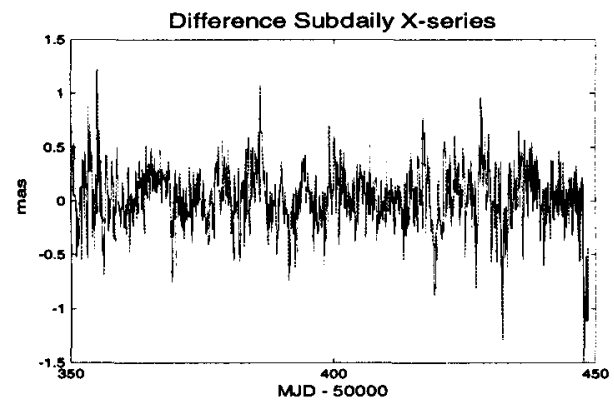

Figure 5. Sub-daily residuals in $\mathrm{x}$.

worse in polar motion $( \pm 150 \mu a s)$, but the short period under consideration should always be kept in mind.

Furthermore, it is quite interesting to compare the new high resolution series to the sub-daily ERPs computed in 1996 (time resolution $2^{h}$, ITRF94). Figures 5 and 6 show the differences for the whole period in PM-x (similar to PM-y) and UT-UTC. After subtracting long periodic systematic effects the series agree in polar motion at the $\pm 200 \mu a s$ level. Obviously, the corresponding series of the axial component is still dominated by a 14-day period. Additionally, there are some very distinct outliers, which might be related to missing tracking data of some of the few ITRF94 defining stations.

Processing of the remaining 1993-1996 GPS data is still under way and the results will be used to replace the corresponding parts of the older series, e.g. in order to evaluate an improved set of ocean tide coefficients.

\section{References}

Beutler, G., M. Rothacher, J. Kouba, R. Weber, This Volume.

Beutler, G., E. Brockmann, W. Gurtner, U. Hugentobler, L. Mervart, M. Rothacher, A. Verdun, 1994, "Extended orbit modeling techniques at the CODE processing center of the IGS: Theory and initial results," Manuscripta Geodaetica, 19, pp. 367-385.

Eanes, R.J., S.V. Bettadpur, 1996, The CSR 3.0 global ocean tide model.

McCarthy, D.D., 1996, IERS Conventions 1996, IERS Technical Note 21, Observatoire de Paris.

Neilan, R.E. (editor), 1998, International GPS Service; IGS Directory, IGS Central Bureau, JPL. 


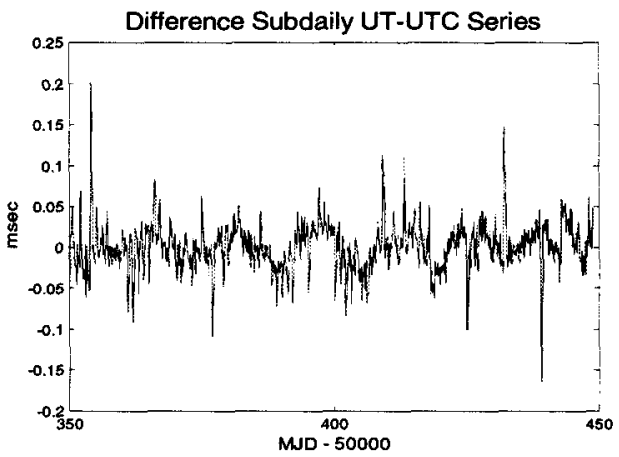

Figure 6. Sub-daily residuals in UT1-UTC.

Rothacher, M., G. Beutler, R. Weber, J. Hefty, 1998, High Frequency Earth Rotation Variations from three Years of GPS data, in Habilitation Thesis, University of Bern, pp. 65-104.

Scherneck, H.G., 1991, A parameterized solid earth tide model and ocean tide loading effects for global geodetic baseline measurements, Geophys. J. Int., 106, pp. 677-694.

Weber, R., 1999, The Ability of the GPS to Monitor Earth Rotation Variations, Acta Geodaetica et Geophysica Hungarica, 34, pp. 457-473. 